\title{
Characteristics of Spontaneously Conceived Twins Versus Twins After Assisted Conception
}

\author{
Ilse Delbaere, Hans Verstraelen, and Marleen Temmerman \\ Department of Obstetrics and Gynaecology, University Hospital Gent, Ghent, Belgium
}

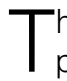
he East Flanders Prospective Twin Survey comprises data concerning placentation, mode of conception and perinatal outcome. As such, this database is convenient to study differences between spontaneous twins and twins resulting from assisted reproductive technologies (ART). Papers based on this survey demonstrated higher incidences of velamentous cord insertion, single umbilical artery and preterm birth in twins after ART.
\end{abstract}

The effect of assisted reproductive technologies (ART) on pregnancy outcome has been extensively studied in recent years. Research on this subject in twins has been particularly challenging in that the absolute majority of nonspontaneously conceived twins is of the dizygotic type. Since monozygotic (MZ) and dizygotic (DZ) twins are not comparable in terms of pregnancy outcome, it is necessary to adjust for zygosity in studies analyzing outcome differences between spontaneously and nonspontaneously conceived twins. As determination of zygosity is not a standard procedure at birth, most researchers include twins of opposite sex only in order to exclude MZ twins. The population-based East Flanders Prospective Twin Survey (EFPTS) is unique in that chorionicity and zygosity have been assessed for all cases. As such, adjustment for these variables is possible which implies that loss of information by excluding an important number of twins can be avoided. The dataset furthermore includes accurate registration of mode of conception with specification of techniques applied in ART. These qualities are apposite to explore differences in characteristics between spontaneously conceived twins and twins after assisted reproduction.

Currently, two studies analyzing differences between spontaneously and nonspontaneously conceived twins are based on EFPTS-data (Delbaere et al., 2007; Verstraelen et al., 2005).

\section{$\overline{\text { Methodology }}$}

For a detailed description of applied methods, we refer to the papers concerned (Delbaere et al., 2007;
Verstraelen et al., 2005). In brief, Verstraelen et al. (2005) compared gestational length and prevalence of preterm birth between 2915 spontaneous twin pairs and 1453 twins born after ART. The 'overall effect' was first assessed by adjustment for pretreatment variables such as birth year, parity and maternal age. Subsequently, mediating effects were removed by adjusting for posttreatment variables including zygosity, chorionicity, intratwin fetal sex and cesarean section.

Delbaere et al. (2007) analyzed differences in cord characteristics between 2119 spontaneously conceived dizygotic twins and 2243 dizygotic twins originating from ART. Data were adjusted for intratwin correlation, year of birth, maternal age, gestational age, parity, sex of the child, number of placentas and type of reproductive technology applied.

\section{Results}

The main findings of Verstraelen et al. (2005) are listed in Table 1 . The overall effect of subfertility treatment on preterm birth was estimated, adjusting for birth year, maternal age, and parity. Subfertility treatment was found to be associated with a small decrease in gestational age at birth compared with the natural conception group. However, these rates were clearly attenuated when accounted for confounding; lower parity in women who conceived artificially was found to be the strongest confounder. Since these confounding effects do not fully cover the observed effect, the direct effect was subsequently estimated by accounting for zygosity, chorionicity, fetal sex and mode of delivery. According to this analysis, dizygotic twinning in nonspontaneous pregnancies proves an advantageous mediator of gestational length as compared to spontaneously conceived twins.

In the Delbaere et al. (2007) study, umbilical cord anomalies were found more frequently in twins originating from ART (see Table 2). The incidence of velamentous insertion doubled in twins conceived by in vitro fertilization and tripled in twins originating from intracytoplasmic sperm injection in comparison with spontaneously conceived twins. Single umbilical artery was found to be more than twice as frequent in 
Table 1

Crude and Adjusted Odds Ratios (95\% Confidence intervals) of Preterm Birth in Ovarian Stimulation and IVF/ICSI Groups of Women, Compared With Natural Conception Group

\begin{tabular}{|c|c|c|c|c|c|c|}
\hline \multirow[b]{2}{*}{ Group } & \multicolumn{2}{|c|}{ Observed effect } & \multicolumn{2}{|c|}{ Overall effect ${ }^{*}$} & \multicolumn{2}{|c|}{ Direct effect ${ }^{\dagger}$} \\
\hline & Odds ratio & $p$ value & Odds ratio & $p$ value & Odds Ratio & $p$ value \\
\hline Natural conception $(n=2915)$ & Reference & - & Reference & - & Reference & - \\
\hline Ovarian stimulation $(n=710)$ & $1.44(1.22-1.70)$ & $<.001$ & $1.26(1.07-1.50)$ & .007 & $1.42(1.18-1.70)$ & $<.001$ \\
\hline IVF/ICSI $(n=743)$ & $1.78(1.51-2.10)$ & $<.001$ & $1.38(1.15-1.66)$ & $<.001$ & $1.58(1.30-1.92)$ & $<.001$ \\
\hline Subfertility treatment $\ddagger(n=1453)$ & $1.61(1.41-1.82)$ & $<.001$ & $1.32(1.14-.51)$ & $<.001$ & $1.49(1.27-1.73)$ & $<.001$ \\
\hline
\end{tabular}

Note: ICSI = intracytoplasmic sperm injection; IVF = in vitro fertilization.

*Multivariate analysis accounting for birth year, maternal age, and parity (confounders).

${ }^{\dagger}$ Multivariate analysis accounting for birth year, maternal age, and parity (confounders) and for infant sex, cesarean delivery, zygosity, and chorionicity (mediating variables).

‡Comprises ovarian stimulation and IVF/ICSI groups.

twins after assisted reproduction compared to spontaneously conceived twins. For this type of cord pathology, artificial induction of ovulation seemed to bring about the greatest risk.

\section{Discussion}

The most important strength of the study by Verstraelen et al. (2005) is the fact that proper adjustment for zygosity and chorionicity was possible, proving the important protective factor of dizygosity in the pregnancy outcome of twins resulting from ART. Preterm birth is nonetheless more frequent in iatrogenic twin pregnancies, apparently largely due to the high amount primiparae in this group.

The results from Delbaere et al. (2007) indicate that artificially conceived dizygotic twins are at high risk for cord anomalies, particularly for velamentous insertion and single umbilical artery. Since cord pathologies, particularly velamentous insertion and single umbilical artery, have been associated with adverse pregnancy outcomes (Heinonen et al., 1996), it would be of interest to adjust for cord anomalies in future studies analyzing pregnancy outcome after ART.

\section{Acknowledgements}

We thank Sylvie Goetgeluk, Catherine Derom and Stijn Vansteelandt for their cooperation.

\section{References}

Delbaere, I., Goetgeluk, S., Derom, C., De Sutter, P., \& Temmerman, M. (2007). Umbilical cord anomalies are more frequent in twins after assisted reproduction. Manuscript submitted for publication.

Heinonen, S., Ryynanen, M., Kirkinen, P., \& Saarikoski, S. (1996). Perinatal diagnostic evaluation of velamentous umbilical cord insertion: Clinical, Doppler, and ultrasonic findings. Obstetrics and Gynecology, 87, 112-117.

Verstraelen, H., Goetgeluk, S., Derom, C., Vansteelandt, S., Derom, R., Goetghebeur, E., \& Temmerman, M. (2005). Preterm birth in twins after subfertility treatment: Population based cohort study. British Medical Journal, 331, 1173.

\section{Table 2}

The Incidence of Central/Eccentric Cord Insertion and Abnormal Umbilical Cord in Dizygotic Twins of the EFPTS — Population 1985-2005, According to Mode of Conception and Adjusted for Maternal Age, Gestational Age, Parity, Year of Birth and Fetal Sex

\begin{tabular}{lccccc}
\hline & $\begin{array}{c}\text { Spontaneous } \\
\text { conception }(n=2119)\end{array}$ & $\begin{array}{c}\text { Artificial induction } \\
\text { of ovulation }(n=912)\end{array}$ & $\begin{array}{c}\text { In vitro fertilization } \\
(n=880)\end{array}$ & $\begin{array}{c}\text { Intracytoplasmic sperm } \\
\text { injection }(n=451)\end{array}$ & $\begin{array}{c}\text { Total assisted } \\
\text { reproductive } \\
\text { technologies }(n=2243)\end{array}$ \\
\hline $\begin{array}{l}\text { Central or eccentric } \\
\text { insertion }\end{array}$ & $1859(87.7 \%)$ & $762(83.6 \%)^{* *}$ & $690(78.4 \%)^{* * *}$ & $356(78.9 \%)^{*}$ & $1808(80.6 \%)^{* * *}$ \\
Marginal insertion & $184(8.7 \%)$ & $104(11.4 \%)^{*}$ & $125(14.2 \%)^{* *}$ & $48(10.6 \%)$ & $277(12.3 \%)^{* * *}$ \\
Velamentous insertion & $76(3.6 \%)$ & $46(5.0 \%)^{+}$ & $65(7.4 \%)^{* * *}$ & $47(10.4 \%)^{* * *}$ & $158(7.0 \%)^{* * *}$ \\
Single umbilical artery & $16(0.6 \%)$ & $22(1.9 \%)^{* * *}$ & $14(1.2 \%)$ & $8(1.2 \%)$ & $44(2.0 \%)^{* * *}$ \\
\hline
\end{tabular}

Note: $p$ values for comparison with spontaneously conceived twins

$$
\begin{aligned}
& { }^{\dagger} \text { Adjusted } p \text { value }=.05 \\
& { }^{*} \text { Adjusted } p \text { value }<.05 \\
& { }^{* *} \text { Adjusted } p \text { value }<.01 \\
& { }^{* * *} \text { Adjusted } p \text { value }<.001
\end{aligned}
$$

